\title{
Análise comparativa da refração automática objetiva e refração clínica
}

\author{
Automatic objective refraction and clinical refraction - a comparative analysis
}

Ricardo Uras ${ }^{(1)}$

Rubens Belfort Jri ${ }^{(2)}$

Ana Luisa Hofling-Lima ${ }^{(3)}$

Elisabeth Nogueira Martins ${ }^{(4)}$

\section{RESUMO}

Objetivo: Este estudo buscou verificar se a prescrição adequada de lentes corretoras pode ser realizada exclusivamente com os dados fornecidos pela refração automática objetiva. Métodos: Todos os pacientes foram submetidos a anamnese, exame oftalmológico. A refração clínica, por meio de recursos propedêuticos clássicos não-automatizados objetivos e subjetivos para prescrição de lentes corretoras ("gold standard"), seguidopor exameno refrator automático TOPCON KR3000. Resultados: Foram estudados 1001 olhos de 504 pacientes, dos quais 45,2\%, do sexo masculino.A média deidadefoide 36,6anos. Oíndicegeralde concordância de diagnóstico entre refração clínica e refração automática objetiva foi de 66,7\%. Considerando-se tolerância de -0,50 a +0,50 DE, o índice de concordância quanto ao componente esférico foi de cerca de $90 \%$. Houve concordância em $27,60 \%$ dos astigmatismos hipermetrópicos e miópicos simples e de $97,7 \%$ nos astigmatismos compostos e no astigmatismo misto. A cicloplegia não alterou de maneira estatisticamente significante oíndice de concordância de diagnóstico. O eixo das lentes cilíndricas indicado pela refração automática objetiva apresentou proximidade estatisticamente significante ao eixo da refração clínica. Conclusão: A refração automática objetiva fornece dados úteis para a prescrição de lentes corretoras, desde que se levem em consideração variáveis como uso prévio ou não de óculos, idade e cicloplegia. A prescrição de lentes corretoras não pode ser realizada exclusivamentecomos dadosfornecidos pela refraçãoautomática objetiva.

Descritores: Lentes de contato; Erros de refração; Refração ocular; Refratometria.

\section{INTRODUÇ̃̃̃O}

A prescrição de lentes corretoras, por tentativa e erro, com lentes de prova, começou há mais de dois séculos. A montagem de lentes em refratores e, posteriormente, em optômetros, diminuiu muito o tempo de execução do exame de refração, sendo o primeiro optômetro descrito em 1937, por Collins, ${ }^{1}$ e modelos aprimorados de refratores continuam a ser introduzidos. A automatização da refração é tentada há várias décadas, porém, apesar dos avanços tecnológicos atuais, ainda não existe o aparelho com precisão absoluta. Os métodos gerais da refração automática baseiamse na óptica da retinoscopia e em dois princípios ópticos básicos há muito conhecidos: o princípio de Scheiner e o princípio do optômetro².

O desenvolvimento da tecnologia eletrônica, a substituição da luz visível pela radiação infravermelha, não-visível para o paciente, e a utilização de microcomputadores para analisar e processar os dados do exame, iniciou 
a era dos refratores automáticos, levando ao surgimento de equipamentos mais rápidos e eficientes ${ }^{3}$.

Nos atuais refratores automáticos, raios infravermelhos dirigidos ao fundo do olho do paciente são refletidos, detectados e analisados por microprocessador que determina a refração objetiva esférica e cilíndrica com respectivo eixo ${ }^{1}$.

O objetivo deste estudo foi verificar se a prescrição adequada de lentes corretoras pode ser realizada exclusivamente com os dados fornecidos pela refração automática objetiva, através da comparação dos resultados refratométricos obtidos pela refração automática objetiva (refrator automático TOPCON NR3000) com os da refração clínica, de acordo com o diagnóstico refracional, o componente cilíndrico, o eixo da lente cilíndrica e o equivalente esférico. A influência do uso prévio de óculos, da idade e da cicloplegia também foram avaliados.

\section{MÉTODOS}

Foram examinados 1.001 olhos de 504 pacientes $(45,2 \%$ de sexo masculino), no período de março de 1994 a julho de 1995 , utilizando-se o ceratorrefrator automático Topcon KR 3000. Este aparelho utiliza uma fonte de luz circular que, refletida na córnea ao redor do eixo visual, determina o raio de curvatura do ápice da córnea em dioptrias e em milímetros (ceratometria automática), além de determinar a refração automática objetiva. Os critérios de inclusão incluíram pacientes com acuidade visual de 20/20 com a melhor correção e exames de refração objetiva, subjetiva, e automática sem dúvidas de interpretação. Pacientes com: opacidades de meios transparentes oculares, acuidade visual com a melhor correção óptica (óculos) menor que 20/20, astigmatismo irregular, ectasia corneana, afacia, pseudofacia, ambliopia, sub-luxação do cristalino ou cirurgia ocular prévia não foram admitidos no estudo.

Todos os pacientes foram submetidos à anamnese detalhada, ectoscopia, biomicroscopia, oftalmoscopia direta, tonometria de aplanação, refração clínica objetiva e subjetiva por meio de recursos propedêuticos não automatizados para prescrição de lentes corretoras (retinoscopia, refrator tipo "Greens" ou armação de prova com lentes avulsas, cilindro cruzado de Jackson e teste bicromático).

Todos os exames foram realizados pelo primeiro autor (RU) que, a seu critério indicou cicloplegia quando o estado dinâmico ocular evidenciou dificuldades para a prescrição das lentes corretoras adequadas por interpretação difícil da retinoscopia, fase subjetiva do exame sem boa colaboração ou pupila pequena.

A cicloplegia foi realizada com colírio de ciclopentolato a $1 \%$, instilado aos zero e cinco minutos e o exame realizado após quarenta minutos da instilação da primeira gota.

Após a refração clínica foram executados os exames no ceratorrefrator automático aparelho TOPCON KR 3000, com mínimo de seis e o máximo de dez medidas, para obtenção da média. $\mathrm{O}$ componente esférico das medidas no refrator automático abrangeu de $+18,00$ dioptrias a $-20,00$ dioptrias e o componente cilíndrico até 7,00 dioptrias, com posição do eixo de $0^{\circ}$ a $180^{\circ}$.
Os resultados do poder dióptrico esférico e cilíndrico foram ajustados para valores múltiplos mais próximos de 0,25 dioptrias, e o componente cilíndrico das refrações registrado no valor negativo. O eixo da lente cilíndrica foi aproximado para os valores múltiplos mais próximos de $5^{\circ}$.

Os diagnósticos das ametropias e da emetropia foram estabelecidos pelos resultados do exame clínico. Para análise da existência do componente cilíndrico detectado pelo refrator automático, considerou-se o valor zero para as ametropias esféricas.

Para a comparação entre os valores estudados, foi utilizado o teste $\mathrm{t}$ de Student (t). Para a análise das tabelas de associação, com variáveis categóricas, utilizou-se o teste do Qui-quadrado $\left(\mathrm{X}^{2}\right)$. Foi definido o nível de significância igual ou menor de $0,05(\alpha=5 \%)$. Os valores de $(p)$ inferiores a 0,05 foram considerados estatisticamente significantes e os menores de 0,001 fortemente significantes.

O protocolo foi submetido e aprovado pela Comissão de Ética da Escola Paulista de Medicina. Todos os pacientes incluídos no estudo foram informados sobre os procedimentos a serem realizados e o caráter voluntário da sua participação neste, sendo obtido o termo de consentimento livre-esclarecido.

\section{RESULTADOS}

A média de idade dos pacientes foi de 36,6 anos, com variação de quatro a 80 anos e $67,3 \%$ dos olhos de pacientes que usavam óculos. Do total dos olhos examinados, 158 foram cicloplegiados $(15,8 \%)$. Na faixa etária até dez anos, a cicloplegia foi realizada em $80 \%$ dos olhos, de 11 a 20 anos em $47 \%$ e de 21 a 30 anos em $20 \%$.

A concordância entre a refração automática objetiva (RAO) e a refração clínica (RC) foi de $66,7 \%$ quanto ao diagnóstico de ametropias e da emetropia. Quando os diagnósticos foram analisados separadamente, as maiores concordâncias ocorreram no astigmatismo miópico composto (AMC) de $95,2 \%$, no astigmatismo hipermetrópico composto (AHC) de $90,1 \%$ e no astigmatismo misto (AM) de 85,0\% (tabela 1 ).

Quanto ao componente esférico das ametropias, a concordância entre a RAO e a RC foi de 32,8\%. Em 71,6\% dos olhos, a variação foi de $-0,25$ a $+0,25$ e em 87,7\%, de - 0,50 a + 0,50D.

Quanto à existência do componente cilíndrico, houve concordância em 27,60\% dos astigmatismos hipermetrópicos e miópicos simples e de $97,7 \%$ nos astigmatismos compostos e no astigmatismo misto $(\mathrm{p}<0,0001)$.

Quanto ao valor do componente cilíndrico, em 34\% dos olhos houve concordância entre a refração automática objetiva e refração clínica $(\mathrm{p}<0,0001)$. O eixo do cilindro, na amostra geral, apresentou concordância em $28,9 \%$ dos olhos $(\mathrm{p}<0,0001)$. Porém, quando o valor do eixo do cilindro foi analisado para cada uma das ametropias com componente cilíndrico, observou-se maior porcentagem de concordância no astigmatismo misto $(45,0 \%)$ e menor, no astigmatismo 


\begin{tabular}{|c|c|c|}
\hline Diagnóstico & $\begin{array}{c}\text { Concordância } \\
\%\end{array}$ & $\begin{array}{c}\text { Discordância } \\
\%\end{array}$ \\
\hline M & 29,6 & 70,4 \\
\hline MAS & 41,1 & 58,9 \\
\hline AMC & 95,2 & 4,8 \\
\hline H & 26,4 & 75,6 \\
\hline AHS & 31,6 & 68,4 \\
\hline AHC & 90,1 & 9,9 \\
\hline AM & 85,0 & 15,0 \\
\hline $\mathbf{E}$ & 10,0 & 90,0 \\
\hline Total & 66,7 & 33,33 \\
\hline \multicolumn{3}{|c|}{$p<0,0001$} \\
\hline \multicolumn{3}{|c|}{ 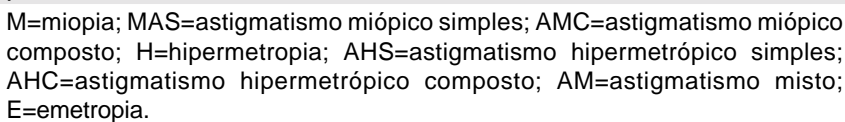 } \\
\hline
\end{tabular}

hipermetrópico simples (21,8\%). Diferenças de 5 a 10 graus foram as mais freqüentes, totalizando $46,5 \%(p<0,191)$.

Houve concordância do equivalente esférico entre RC e RAO em 30,6\% dos olhos (tabela 2).

$\mathrm{Na}$ comparação entre RAO e RC, quanto ao equivalente esférico em cada um dos diagnósticos, observou-se em 53,5\% dos olhos discordância entre 0,25 e 0,50D. Diferenças maiores que 1,00D não foram observados nos AHS ou nos emétropes $(\mathrm{p}<0,048)$.

Entre os 673 pacientes com uso prévio de óculos, a concordância do diagnóstico foi de $77,4 \%$ e nos não usuários de óculos foi de $44,8 \%(\mathrm{p}<0,0001)$.

Já em relação ao componente esférico das ametropias miópicas (miopia e astigmatismo miópico composto), a concordância entre a RAO e a RC foi $38,0 \%$ neste grupo e $31,7 \%$ nos não-usuários de óculos $(\mathrm{p}<0,0001)$.

Em relação ao componente esférico das ametropias hipermetrópicas (hipermetropia e astigmatismo hipermetrópico composto), a concordância foi 34,3\% nos usuários de óculos e $27,9 \%$ nos não-usuários ( $\mathrm{p}<0,014)$.

Quanto ao valor do componente cilíndrico das ametropias cilíndricas (astigmatismo miópico simples, astigmatismo miópico composto, astigmatismo hipermetrópico simples, astig-

\begin{tabular}{|cc|}
\hline $\begin{array}{c}\text { Tabela 2. Discordância entre refração clínica e refração automá- } \\
\text { tica objetiva quanto ao equivalente esférico em dioptrias. }\end{array}$ & $\%$ \\
Diferença entre RC e RAO & 1,8 \\
$\mathrm{RC}<\mathrm{RAO}>$ de 1,00 & 3,9 \\
$\mathrm{RA}<\mathrm{RAO} 0,75$ a 1,00 & 24,2 \\
$\mathrm{RC}<\mathrm{RAO} 0,25$ a 0,50 & 30,6 \\
$\mathrm{RC}=\mathrm{RAO}$ & 29,4 \\
$\mathrm{RC}>\mathrm{RAO} 0,25$ a 0,50 & 6,7 \\
$\mathrm{RC}>\mathrm{RAO} 0,75$ a 1,00 & 3,5 \\
$\mathrm{RC}>\mathrm{RAO}>$ de 1,00 & $\mathbf{1 0 0 , 0}$ \\
Total & \\
p=0,097 & \\
$\mathrm{RC}=$ refração clínica; $\mathrm{RAO}=$ refração automática objetiva. &
\end{tabular}

matismo hipermetrópico composto e astigmatismo misto), a concordância nos usuários de óculos foi $34,2 \%$ e nos nãousuários, 33,5\% ( $<<0,022)$. Quanto ao eixo, a comparação mostrou concordância em $31,8 \%$ nos usuários e $20,0 \%$ nos nãousuários $(\mathrm{p}<0,048)$.

Em relação ao equivalente esférico houve concordância de $28,2 \%$ nos usuários de óculos e de $35,4 \%$ nos não-usuários (tabela 3).

A análise da influência da idade nos resultados mostrou concordância de diagnósticos (ametropias e emetropia) entre a RC e a RAO, na faixa etária até 30 anos, em $60,1 \%$ e na faixa etária de 31 a 80 anos em $71 \%(\mathrm{p}<0,0001)$. Quando os diagnósticos foram analisados separadamente, os maiores índices de concordância foram encontrados nos astigmatismos miópicos compostos, nos astigmatismos hipermetrópicos compostos e nos astigmatismos mistos, tanto na faixa etária até 30 anos quanto na faixa etária de 31 a 80 anos.

Dividindo a amostra em quatro faixas etárias, a saber, de 0 a 20 anos, de 21 a 40 anos, de 41 a 50 anos e de 51 a 80 anos, os resultados estão expressos na tabela 4 .

Quando o componente esférico das ametropias foi analisado, dividindo-se a amostra nas faixas etárias: inferior a 30 anos e de 31 a 80 anos, a concordância na primeira faixa foi de $29,9 \%$ e na segunda, $34,6 \%$. A discordância de 0,25 a $0,50 \mathrm{D}$ na faixa etária inferior a 30 anos foi observada em $52,9 \%$ e na faixa etária de 31 a 80 anos em $56,2 \%$ ( $<<0,0003)$.

A comparação entre RAO e RC, quanto ao valor do componente cilíndrico, apresentou concordância de $29,9 \%$ na faixa etária inferior a 30 anos e de $37,0 \%$ na faixa etária de 31 a 80 anos. Discordâncias de 0,25 e 0,50D ocorreram em 59,9\% dos olhos na faixa etária de 0 a 30 anos e $54,9 \%$ na faixa etária de 31 a 80 anos.

\begin{tabular}{|lcc|}
\hline \multicolumn{3}{|c|}{$\begin{array}{c}\text { Tabela 3. Porcentagens de discordância do equivalente } \\
\text { esférico em dioptrias entre a refração clínica e refração }\end{array}$} \\
automática objetiva em usuários e não-usuários de óculos \\
Diferenças do equivalente & Usuários & Não-usuários \\
esférico entre a RC e RAO & $\%$ & $\%$ \\
$\mathbf{0 , 0 0}$ & 28,2 & 35,4 \\
$\mathbf{0 , 2 5}$ a $\mathbf{0 , 5 0}$ & 55,0 & 50,6 \\
$\mathbf{0 , 7 5}$ a $\mathbf{1 , 0 0}$ & 10,5 & 10,7 \\
>1,00 & 6,3 & 3,3 \\
TOTAL & $\mathbf{1 0 0 , 0}$ & $\mathbf{1 0 0 , 0}$ \\
P = 0,047 & \\
RC=refração clínica; RAO=rafração automática objetiva. \\
\hline
\end{tabular}

\begin{tabular}{|c|c|c|c|c|}
\hline & $\begin{array}{c}0-20 \\
\%\end{array}$ & $\begin{array}{c}21-40 \\
\%\end{array}$ & $\begin{array}{c}41-50 \\
\%\end{array}$ & $\begin{array}{c}51-80 \text { anos } \\
\%\end{array}$ \\
\hline Concordância & 54,4 & 64,8 & 74,3 & 74,1 \\
\hline Discordância & 45,6 & 35,2 & 25,7 & 25,9 \\
\hline TOTAL & 100,0 & 100,0 & 100,0 & 100,0 \\
\hline$p<0,0001$ & & & & \\
\hline
\end{tabular}


A avaliação quanto ao eixo do cilindro nestas faixas etárias apresentou concordância de $26,9 \%$ e $30,1 \%$, respectivamente.

Quanto ao equivalente esférico houve concordância de $27,9 \%$ no subgrupo inferior a 30 anos e de $32,3 \%$ no subgrupo de 31 a 80 anos (tabela 5).

Já a comparação entre os resultados da RAO e da RC nos pacientes cicloplegiados e nos não-cicloplegiados, mostrou porcentagem de concordância dos diagnósticos de 59,0\% nos olhos cicloplegiados e $60,9 \%$ nos não cicloplegiados ( $\mathrm{p}<0,847$ ).

Ao comparar a RC e RAO quanto ao componente esférico, incluindo todas as ametropias e a emetropia, a concordância do componente esférico nos pacientes cicloplegiados foi $37,0 \%$ e nos não-cicloplegiados, $25,7 \%(\mathrm{p}<0,0008)$.

A análise comparativa do componente esférico das ametropias hipermetrópicas (hipermetropia e astigmatismo hipermetrópico composto) mostrou concordância de 32,6\% nos olhos cicloplegiados e de $27,9 \%$ nos olhos não-cicloplegiados. Nas ametropias miópicas (miopia e astigmatismo miópico composto) mostrou concordância de $44,1 \%$ nos cicloplegiados e $29,8 \%$ nos não-cicloplegiados.

Quanto ao componente cilíndrico, nos olhos não-cicloplegiados, houve concordância em 25,3 e nos olhos cicloplegiados, de 35,6\%.

$\mathrm{Na}$ comparação entre os resultados da RC e da RAO nas ametropias com componente cilíndrico, a concordância no eixo do cilindro foi $28,8 \%$ nos não-cicloplegiados e $24,4 \%$ nos cicloplegiados. A diferença de 5 a 20 graus nos olhos nãocicloplegiados foi observada em $62,0 \%$ e $27,8 \%$ nos cicloplegiados $(\mathrm{p}<0,19)$.

Em relação ao equivalente esférico houve concordância de $22,9 \%$ nos olhos não-cicloplegiados e de $36,30 \%$ nos cicloplegiados (tabela 6).

\section{DISCUSSÃO}

Existe uma tendência de equipar consultórios oftalmológicos com refratores automáticos, acreditando-se que possam ser bons auxiliares do oftalmologista na prescrição de lente corretoras. O fascínio provocado pelos aparelhos computadorizados, de maneira geral, deixa ao leigo e a alguns médi-

\begin{tabular}{|c|c|c|}
\hline \multicolumn{3}{|c|}{$\begin{array}{l}\text { Tabela 5. Porcentagens de discordância do equivalente } \\
\text { esférico entre a refração clínica e a refração automática } \\
\text { objetiva em olhos de pacientes nas faixas etárias até } 30 \text { e de } \\
31 \text { a } 80 \text { anos }\end{array}$} \\
\hline $\begin{array}{l}\text { Diferenças em dioptrias } \\
\text { Esféricas entre RC e RAO }\end{array}$ & $\begin{array}{c}\text { Até } 30 \text { anos } \\
\%\end{array}$ & $\begin{array}{c}\text { De } 31 \text { a } 80 \text { anos } \\
\%\end{array}$ \\
\hline 0,00 & 27,9 & 32,3 \\
\hline 0,25 a 0,50 & 52,7 & 54,1 \\
\hline 0,75 a 1,00 & 13,8 & 7,4 \\
\hline$>1,00$ & 5,6 & 6,2 \\
\hline TOTAL & 100,0 & 100,0 \\
\hline \multicolumn{3}{|l|}{$p=0,008$} \\
\hline $\mathrm{RC}=$ refração clínica; $\mathrm{RAO}=$ refrac & o automática obj & \\
\hline
\end{tabular}

\begin{tabular}{|lcc|}
\hline \multicolumn{3}{|c|}{$\begin{array}{c}\text { Tabela } \\
\text { 6. Porcentagens de discordância do equivalente } \\
\text { esférico nos olhos cicloplegiados e nos } \\
\text { não-cicloplegiados }\end{array}$} \\
$\begin{array}{l}\text { Diferenças do } \\
\text { componente }\end{array}$ & $\begin{array}{c}\text { Cicloplegiados } \\
\%\end{array}$ & $\begin{array}{c}\text { Não-cicloplegiados } \\
\%\end{array}$ \\
$\mathbf{0 , 0 0}$ & 36,3 & 22,9 \\
$\mathbf{0 , 2 5}$ a $\mathbf{0 , 5 0}$ & 55,5 & 51,0 \\
$\mathbf{0 , 7 5}$ a $\mathbf{1 , 0 0}$ & 7,5 & 17,6 \\
> 1,00 & 0,7 & 8,6 \\
Total & $\mathbf{1 0 0 , 0}$ & $\mathbf{1 0 0 , 0}$ \\
$\mathrm{p}=0,0001$ & & \\
\hline
\end{tabular}

cos, a impressão que o resultado é infalível. Este trabalho procurou conhecer e entender a real utilidade e as restrições do refrator automático, a fim de evitar que este seja superestimado, em detrimento do raciocínio e do conhecimento.

O aparelho utilizado neste estudo foi o TOPCON KR 3.000, usado em pacientes nos quais o único problema ocular era o erro de refração (olhos normais) e onde ambos os procedimentos (clínico e convencional) não deixaram dúvidas quanto à interpretação.

$\mathrm{Na}$ análise geral da amostra, observou-se que a maior concordância do diagnóstico do erro de refração aconteceu no AMC (95,2\%), seguido do AHC (90,1\%) e do AM (85,0\%). O menor grau de concordância na comparação entre RAO e RC, quanto ao diagnóstico, foi emetropia $(10,0 \%)$. Observou-se em $72,4 \%$ dos olhos, um cilindro onde não havia. Já nas ametropias cilíndricas, apenas em $2,3 \%$ dos casos a RAO deixou de detectar componente cilíndrico. Estes dados podem ser explicados pela tendência de os refratores automáticos sugerirem cilindros de pequeno valor em olhos emétropes, levando, por esse motivo, ao reduzido número de diagnósticos de emetropia ${ }^{4}$.

Ainda na análise geral, a concordância do componente esférico entre RC e RAO foi 32,8\%, sendo observada uma variação de -0,25DE a +0,25DE em 71,6\%.

Quanto ao valor do componente cilíndrico, ficou demostrado que em $34 \%$ dos casos houve concordância entre a RAO e a RC. Variações de $\pm 0,25 \mathrm{DC}$ e de $\pm 0,50 \mathrm{DC}$ ocorreram em $77,7 \%$ e $92,8 \%$, respectivamente, em concordância com a literatura $^{5-9}$.

Quando foi analisado o eixo do cilindro, verificou-se que o indicado pelo refrator automático é próximo da refração clínica. Houve concordância em 28,9\% dos casos, sendo que diferenças até $5^{\circ}$ e até $10^{\circ}$ ocorreram em $57,7 \%$ e $75,4 \%$, respectivamente. Quando a análise foi feita para cada uma das ametropias cilíndricas, foi no astigmatismo misto que ocorreu a maior porcentagem $(45,0 \%)$ de concordância do eixo do cilindro. O maior acerto da RAO na determinação do eixo do cilindro no astigmatismo misto já foi descrito anteriormente ${ }^{4}$, sendo este acerto mais freqüente quanto maior o valor do cilindro ${ }^{6,10-11}$.

$\mathrm{O}$ equivalente esférico, quando analisado no total da amostra, mostrou uma concordância de 30,6\% entre RC e RAO. Quando esta análise foi feita para cada uma das 
ametropias, demostrou-se que a menor concordância foi encontrada no AM (29,3\%) e a maior no AHC (34,0\%). Apesar de o equivalente esférico, às vezes, não retratar o real estado refracional do olho, vários trabalhos científicos, ao estudar refração, utilizam esse parâmetro.

A influência do uso de óculos nos resultados da RAO também foi analisada. Quanto ao diagnóstico, houve uma concordância maior nos usuários de óculos. O componente esférico, quando analisado nas ametropias miópicas (M-AMC) e hipermetrópicas ( $\mathrm{H}$ - AHC) demonstrou que a determinação do componente esférico, tanto negativo quanto positivo, também é mais concordante nos usuários de óculos. Já na análise do componente cilíndrico não foi demostrada que a determinação do cilindro pela RAO pudesse ser influenciada pelo uso prévio ou não de óculos, porém a concordância do eixo do cilindro nos usuários de óculos foi maior. Com relação ao equivalente esférico, nos não-usuários de óculos, a concordância foi maior.

A influência do uso ou não de óculos não foi estudada em nenhum dos trabalhos pesquisados sobre refração automática. O emétrope que usa correção óptica e que já está adaptado ao uso de lentes corretoras, usando menos a acomodação e, eventualmente, mais familiarizado com o ambiente e com a rotina do exame de refração, talvez leve à maior concordância entre RAO e RC.

Com relação à idade, demonstrou-se que, nos olhos dos pacientes na faixa etária até trinta anos, a concordância no diagnóstico entre RAO e RC foi de $60,0 \%$ e de $71,0 \%$ na faixa de trinta e um a oitenta anos. Analisando a concordância ou não de diagnóstico nas duas faixas etárias para cada ametropia e para a emetropia, não houve diferença entre os grupos estudados. Porém, quando a amostra foi dividida em quatro faixas etárias (até 20 anos, de 21 a 40 anos, de 41 a 50 anos e de 51 a 80), verificou-se um aumento progressivo de concordância de diagnóstico da primeira para a terceira faixa, e uma estabilidade entre a terceira e a quarta faixas etárias, sugerindo a acomodação visual como causa desta diferença.

A análise do componente esférico das ametropias mostrou, na faixa etária até trinta anos, concordância de 29,2\%, e de $37,0 \%$ na faixa de 31 a 80 anos. Mais uma vez, parece que, quanto menor o potencial de acomodação dos pacientes, maior a precisão da refração automática, fato também observado anteriormente ${ }^{4,13}$. Sabe-se também que existe uma tendência de a refração automática miopizar olhos, principalmente nos pacientes com menos de 20 anos 4, 13-14.

A influência da idade na determinação do eixo do componente cilíndrico nas faixas etárias até 30, e de 31 a 80 anos não foi significativa. A margem de concordância entre RC e RAO, para uma diferença de até $10^{\circ}$, foi $75,9 \%$ e $75,2 \%$ na primeira e na segunda faixa etárias, respectivamente. A comparação do equivalente esférico entre a RC e a RAO nas faixas etárias analisadas mostrou que nos mais jovens, a concordância é menor.

Pela constatação que, em usuários de óculos e em pessoas de mais idade, o resultado da refração automática é mais concordante com a refração clínica e que provavelmente isto se deva ao esforço acomodativo ser menor em ambos os casos, impõe-se a avaliação do efeito da cicloplegia nos resultados comparativos da RAO e da RC.

Assim, para avaliar a influência da cicloplegia na refração automática, foram estudados os 391 olhos dos pacientes com até 30 anos de idade. Quando comparou-se o diagnóstico das refrações estática e dinâmica entre a RAO e a RC verificou-se, de maneira geral, que a cicloplegia não exerce influência significativa sobre o diagnóstico. Quando analisou-se a influência da cicloplegia no valor do componente esférico, foi significativa a constatação de que nos cicloplegiados, o exame com o refrator automático é mais concordante (cicloplegiados, 37,0\% e não-cicloplegiados, $25,7 \%$ ).

Analisando o componente esférico nas ametropias, também foi constatado, de maneira significativa, que nos olhos cicloplegiados, a concordância entre a RAO e a RC é maior do que nos olhos não-cicloplegiados.

Nos olhos com ametropias hipermetrópicas, as hipocorreções ocorreram em 40,8\% nos não-cicloplegiados, e em 23,9\% nos ciloplegiados. Nos olhos com ametropias miópicas (M e AMC), as hipercorreções aconteceram em 41,7\% nos nãocicloplegiados, e em 22,1\% nos cicloplegiados. A comparação mostra que a refração automática tende a hipercorrigir míopes e a hipocorrigir hipermétropes, principalmente se o exame for realizado no estado dinâmico do olho.

Analisando a influência da cicloplegia na intensidade do componente cilíndrico, apesar da maior porcentagem de acerto nos cicloplegiados, não ser estatisticamente significante, houve melhora da precisão da refração automática na determinação do cilindro com a cicloplegia. Quanto à posição do eixo do cilindro, também não se observou que a cicloplegia pudesse ter efeito significativamente favorável no exame automatizado. Observou-se que diferenças até 0,50 dioptrias do componente cilíndrico, tanto nos cicloplegiados como nos nãocicloplegiados, ocorreram em cerca de $90 \%$ dos olhos. Para a posição do eixo do cilindro, verificou-se que em aproximadamente $75 \%$ dos olhos cicloplegiados ou não, as diferenças foram de até $10^{\circ}$. A importância da cicloplegia na refração automática também é reconhecida na literatura ${ }^{10,13,15-16}$.

A análise do equivalente esférico das refrações nos pacientes cicloplegiados e nos não-cicloplegiados mostrou a significância da cicloplegia como fator favorável para o exame automatizado. Este fato foi também observado em estudos em crianças ${ }^{17-18}$.

Com o avanço da tecnologia, a tendência para a informatização é irreversível. No caso dos refratores automáticos, porém, é preciso ter cautela com relação ao seu uso indevido, conhecendo suas vantagens e limitações.

Os refratores automáticos representam importantes auxiliares do oftalmologista na prescrição de lentes corretivas, sem, contudo, poder substituí-lo. Em alguns, casos, como no exame de crianças, desde que devidamente cicloplegiadas é 
indiscutível sua utilidade, porque geralmente as respostas dos pacientes, não são confiáveis ou são inexistentes.

O tempo de exame para o paciente obter a sua prescrição é menor quando usamos os refratores automáticos, devido, principalmente, ao fato de que o exame clínico objetivo, em alguns casos, pode deixar de ser realizado. Porém, é no exame subjetivo que acontece a maior interação entre o paciente e o oftalmologista que, assim, colhe o maior número de informações possível, o que possibilitará a adequada prescrição de lente corretoras.

A indicação de cicloplegia em pacientes com idade até dez anos $(80 \%)$, e entre 11 e 20 anos (47\%), foi menor do que a descrita em outros estudos ${ }^{19-20}$. Com a utilização de critérios clínicos a necessidade de cicloplegia é dependente da experiência do examinador. Ressalta-se, porém que não se deve preconizar este baixo índice, e sim a sua realização em todos os pacientes em que haja influência importante da acomodação na refração dinâmica.

\section{CONCLUSÕES}

Este trabalho objetivou comparar os resultados da refração clínica, executada pelo autor, com os da refração automática objetiva (Topcon KR3000) em 1.001 olhos normais, avaliando a influência das variáveis idade, uso prévio ou não de óculos e cicloplegia, comparando diagnóstico, componente esférico, componente cilíndrico, eixo do cilindro e equivalente esférico.

Pela análise dos resultados, observou-se que algumas das variáveis estudadas exercem influência sobre o exame automatizado. A conclusão do estudo é que o refrator automático objetivo pode ser um instrumento útil para o oftalmologista como auxiliar na prescrição de lentes corretoras, sem fornecer, porém, dados suficientemente exatos, a ponto de eliminar a necessidade da refração subjetiva.

\section{ABSTRACT}

Purpose: This study was designed to determine if lens prescription can be based solely on automated objective refraction. Methods: All patients were interviewed and underwent an ophthalmologic examination including clinical refraction with classical non-automated objective and subjective procedures (gold standard). Afterwards the automated objective refraction was performed using the automatic keratorefractor TOPCON 3000. Results: 1,001 eyes of 504 patients were studied. $45.2 \%$ were male patients and the mean age was 36.6 years. There was an overall concordance between clinical refraction and the automated objective refraction in $66.7 \%$ of the patients. The concordance of a spherical value, not considering variations of -0.50 to $+0.50 \mathrm{SD}$ was, approximately $90 \%$. In simple hyperopic/myopic astig-matic eyes the concordance was $27.6 \%$, in eyes with compound hyperopic/myopic astigmatism the concordance was $97.7 \%$. Cycloplegia did not significantly affect this concordance. There was no significant difference regarding the axis of astigmatic eyes when using both techniques. Conclusion: Automated objective refraction is an useful tool in clinical refraction but clinical data should also be considered and the final lens prescription should never be based solely on the automated examination.

Keywords: Contact lenses; Refractive errors; Ocular refraction; Refractometry.

\section{REFERÊNCIAS}

1. Wood IC. A review of autorefractors. Eye 1987;1:529-35.

2. Edwards K, Lewellyn R. Optometria. Barcelona: Cientificas e Tecnicas, 1993.

3. Benchimol R. Refração automatizada. In: Alves AA, editor. Refração. Rio de Janeiro: Cultura Médica; 1994. p. 166-74.

4. Mohan M, Verma L, Kalra VK, Jain A, Kumar H. Accuracy of automated refractors: experience with Nikon AR $1000 \mathrm{~F}$ autorefractor. Indian J Ophthalmol 1987;35:411-5.

5. Poletti J, Courrejou C. Interet et fiabilite d'un refractometre objectif automatique: le dioptron nova. Etude statistique sur ordinateur de 600 mesures. Bull Soc Ophthalmol Fr 1985;4:485-7.

6. Wesemann W, Rassow B. Automatic infrared refractors: a comparative study. Am J Optom Physiol Opt 1987;64:627-38.

7. Carvalho MVM. Avaliação clínica do autorefrator objetivo em pseudofácicos. Rev Bras Oftalmol 1990;49:63-9.

8. Raj PS, Villada JR, Myint K, Lewis AE, Akingbehin T. Clinical evaluation of automated refraction in anterior chamber pseudophakia. Br J Ophthalmol 1991;75:42-4.

9. Raj PS, Villada JR, Lewis AE, Joyce PS, Watson A. Comparative evaluation of the Allergan Humphrey 570 and Canon RK-1 autorefractors: I. Objective autorefraction in normal subjects. Eye 1992;6:284-6.

10. Holtmann H, Kroll P. Ergebnisse mit einem automatischen Refraktometer. Klin Monatsbl Augenheilkd 1975;166:532-6.

11. Semeghini AT, Kamei SC, Wakisaka E. Avaliação clínica do refrator automático Nidek A.R. 1.1100. Arq Bras Oftalmol 1988;51:91-4.

12. Verboven L, Missoten L. Possibilities and limitations of refraction measurement by an automatic refractor. Bull Soc Belge Ophthalmol 1982;201:65-70.

13. Dufier JL, Abitbol M, Pigamo F, Prete T, Paris JP, Poitrenaud O. Evaluation des performances d'un réfractométre objectif automatique sur 3618 yeux. J Fr Ophtalmol 1987;10:301-8.

14. Ghose S, Nayak BK, Singh JP. Critical evaluation of the NR-1000F autorefractometer. Br J Ophtalmol 1986;70:221-6.

15. Salvesen S, Kohler M. Precision in automated refraction. Acta Ophthalmol (Copenh) 1991;69:338-41.

16. Quere MA, Lavenant F, Milazzo M, Chadan N, Joly P. Amblyopiestrabisme-nystagmus. Apport de la refractometrie automatique sans leur correction optique. Bull Soc Ophthalmol Fr 1988;88:1301-4.

17. Hunold W, Auffarth G, Effert R. Klinische anwendung des Canon Aurefraktometers R10 zur refraktionsbestimmung bei schiekjindern. Klin Monatsbl Augenheilkd, 1988;192:58-65.

18. Reddy NS, Agarwal S. Clinical evaluation of Topcon auto refractometer. Indian J Ophthalmol 1987;35:407-10.

19. Harvey EM, Miller JM, Wagner LK, Dobson V. Reproducibility and accuracy of measurements with a hand held autorefractor in children. Br J Ophthalmol 1997;81:941-8.

20. El-Defrawy S, Clarke WN, Belec F, Pham B. Evaluation of a hand-held autorefractor in children younger than 6. J Pediatr Ophthalmol Strabismus 1998;35:107-9. 